\title{
Inter- and intra-observer reliability of clinical movement-control tests for marines
}

\author{
Andreas Monnier ${ }^{1,2}$, Joachim Heuer ${ }^{3}$, Kjell Norman $^{4}$ and Björn O Äng ${ }^{1 *}$
}

\begin{abstract}
Background: Musculoskeletal disorders particularly in the back and lower extremities are common among marines. Here, movement-control tests are considered clinically useful for screening and follow-up evaluation. However, few studies have addressed the reliability of clinical tests, and no such published data exists for marines. The present aim was therefore to determine the inter- and intra-observer reliability of clinically convenient tests emphasizing movement control of the back and hip among marines. A secondary aim was to investigate the sensitivity and specificity of these clinical tests for discriminating musculoskeletal pain disorders in this group of military personnel.

Methods: This inter- and intra-observer reliability study used a test-retest approach with six standardized clinical tests focusing on movement control for back and hip. Thirty-three marines (age $28.7 \mathrm{yrs}$, SD 5.9) on active duty volunteered and were recruited. They followed an in-vivo observation test procedure that covered both low- and high-load (threshold) tasks relevant for marines on operational duty. Two independent observers simultaneously rated performance as "correct" or "incorrect" following a standardized assessment protocol. Re-testing followed 7-10 days thereafter. Reliability was analysed using kappa ( $\mathrm{k}$ ) coefficients, while discriminative power of the best-fitting tests for back- and lower-extremity pain was assessed using a multiple-variable regression model.

Results: Inter-observer reliability for the six tests was moderate to almost perfect with $\mathrm{k}$-coefficients ranging between 0.56-0.95. Three tests reached almost perfect inter-observer reliability with mean $\mathrm{k}$-coefficients $>0.81$. However, intra-observer reliability was fair-to-moderate with mean $\mathrm{k}$-coefficients between $0.22-0.58$. Three tests achieved moderate intra-observer reliability with $\mathrm{k}$-coefficients $>0.41$. Combinations of one low-and one high-threshold test best discriminated prior back pain, but results were inconsistent for lower-extremity pain.

Conclusions: Our results suggest that clinical tests of movement control of back and hip are reliable for use in screening protocols using several observers with marines. However, test-retest reproducibility was less accurate, which should be considered in follow-up evaluations. The results also indicate that combinations of low- and high-threshold tests have discriminative validity for prior back pain, but were inconclusive for lower-extremity pain.
\end{abstract}

Keywords: Military, Motor control, Reproducibility, Screening, Sensitivity, Specificity, Validity

\section{Background}

Musculoskeletal disorders, especially in back and lower extremities, are common in marines [1,2] both during their basic military training [3] and later during service [4]. For marines, this could, as for many other military branches [5], reduce their operational efficiency and end their service prematurely $[4,6-8]$. Since it appears common that such problems lead to shift changes, the use of back-up personnel [5] or increased workloads for

\footnotetext{
* Correspondence: Bjorn.Ang@ki.se

${ }^{1}$ Department of Neurobiology, Care Sciences and Society, Division of

Physiotherapy, Karolinska Institutet, Huddinge, Sweden

Full list of author information is available at the end of the article
}

remaining personnel, one marine's disorders could affect the operational efficiency of an entire unit. Back and lower-extremity disorders have in addition been found to be a major contributor to reduction of marine unit strength before deployment, due to medical downgrading of the sufferers to non-deployment status [4]. Further, during deployment, musculoskeletal disorders are the most common causes of medical evacuation $[9,10]$ and marines that suffer incidents of musculoskeletal disorder or spinal pain show less than $20 \%$ likelihood of returning to operational duty [10]. Early physical screening tests focusing on recruits' musculoskeletal health

\section{Biomed Central}


and function in relation to military duty are commonly used in modern armed forces. In the literature, however, no data on reliability exists on such clinical tests in marines.

Several studies in civilian populations have demonstrated a link between musculoskeletal disorders, pain and the ability to adequately control movements and muscular activation in clinical tests [11-13]. Some of these clinical tests are designed for focusing on movement control of a certain defined body region whilst actively moving an adjacent one. Such tests of movement control, also referred to in the literature as motorcontrol tests [14-16], low-load [15] or low-threshold [17] movement-control tests, are suggested for identifying deficits associated with repetitive low-load activity or static positioning [17]. It is suggested that such nonfatiguing movement-control tests will predominantly recruit slow motor units activated at a low threshold [17]. Clinical tests that on the other hand include high load or speed will involve recruitment of fast motor units [17], which are thus activated at a higher threshold and are less resistant to fatigue [18]. These high-threshold tests have therefore been suggested for identifying the risk of injuries in activities involving fatiguing or repeated high loads [17]. In our experience, based on clinical findings and empiric field observation with marines, tests covering low- and high-threshold movement control of the lower back and hip may adequately challenge weak-links in marines' musculoskeletal system as relevant in their operative duty. We believe such assessments to be suitable to include in protocols screening for deficits that may relate to musculoskeletal disorders, induced by exposures from various work tasks or postures in marines. Therefore, in this study, the tests included were selected to evaluate marines' ability to control or prevent defined movements of the lumbar spine and hip while performing specific lower extremity movement.

However, as the results of clinical testing may influence the testee/s future service or career, e.g. by possibly resulting in medical downgrading, it is of great importance for the tests to be reliable and valid for the specific group and its purpose. Specifically, since screening tests for military purposes are commonly used by multiple testers, good inter-observer reliability is required. If the test is to be used with follow-up evaluations, it needs also to show good intra-observer reproducibility [19]. Here, three important aspects influence measurement variability: variation related 1) to the observer(s), 2) to the instrument and the measuring procedure, and 3) to the subject tested [20].

Further, a clinically convenient test should show good validity, i.e. measure the entity that it purports to measure [20]. Specifically, evidence of discriminative validity is required to justify the use of clinical tests of musculoskeletal pain, i.e. how far the tests are able to differentiate between those with back- and lower-extremity pain and those without. Such testing accuracy may complement simple pain ratings, particularly in the work with early recognition of disorders, and possibly for planning further clinical examination and intervention. Useful clinical tests that aim to screen marines' physical function need, at the same time, to be simple and fairly brief since generally many personnel are being tested. In addition, methodological evaluations of clinical tests should advantageously be contingent on clinical contextual factors that reflect the testees' natural environment. Although clinical experience suggests that findings of impaired movement control relate to musculoskeletal disorders and pain episodes in the back and lower extremities, we have found no studies on movementcontrol tests that address such discriminative validity. Further, a few studies in civilian populations (subjects with back pain, subjects with musculoskeletal pain but not back pain and healthy controls) have evaluated the reliability of movement-control tests for the lower back and/or extremities, with inter-tester reliability ranging from poor to almost perfect/excellent [14,15,21-23], and intra-tester reliability from fair to excellent [21]. However, to our knowledge, there is no such published data on reliability of clinical tests in marines. The present aim was therefore to determine the inter- and intraobserver reliability of clinically convenient tests for assessing movement control of back and hip in marines. A secondary aim was to investigate the discriminative validity of the best fitting combination of tests for identifying back and lower-extremity pain disorders in this group of military personnel.

\section{Methods}

\section{Study design}

This inter- and intra-observer reliability study used a test-retest approach and in-vivo testing methodology. The study protocol included six standardized clinical tests that emphasize active movement control for back and hip. Performances were scored simultaneously by two, well-experienced physiotherapists (observers) who were familiar with the tests. The observers were blinded to each other's scores and to the subjects' health and background information. The procedure was repeated 7-10 (mean $=7.4)$ days thereafter. The six tests were to be assessed as "correct" (pass) or "incorrect" (fail), thus generating binominal data. Based on this, the sample size was calculated to approximately 34 subjects at a presumed agreement of $90 \%$ (CI 20\%; chance agreement: $50 \%$ ) [24] and enrolment was planned to meet this criterion. Written informed consent was obtained from all subjects, who received both written and oral information 
prior to participation. Confidentiality and voluntary participation were strongly stressed. The study was approved in advance by the Regional Medical Research Ethics Committee, Stockholm.

\section{Study sample}

Thirty-three marines on active duty (assault infantry, combat craft crews and coastal rangers) were recruited from a combined company of the 2nd Amphibious Battalion, 1st Marine Regiment, Berga, Sweden, the main marine regiment in Sweden. Eligible subjects had to be in service during the test period. Excluded were subjects on limited duty due to illness (full- or part-time sick leave) and subjects temporarily posted or under training at the 2nd Amphibious Battalion. After receiving oral and written information, volunteering subjects were enrolled in the study and scheduled for testing. Of the 33 subjects enrolled, 32 were male and one female. Means (SD) for age, weight and height were: 28.7 (5.9) yrs, 82.5 (9.4) kg and $1.81(0.059) \mathrm{m}$.

\section{Subjective measures of back and lower extremity pain}

Standardized self-report questionnaires were used to collect demographic information and medical history for the previous six months, including numerical rating scales of 'pain at present' and for 'the previous six months' [25], specified by anatomical body region [26]. The numerical pain-rating scale has been found reliable and sensitive for the assessment of pain and has been suggested to be appropriate for use in clinical practice [25] and research [27]. For the purpose of this study, back- (lumbar, thoracic-back) and lower-extremity (hip/ thigh, knee, ankle/foot regions) pain was defined as any reported pain experience (pain, ache or discomfort), and for pain at present this was $\geq 1$ on the numerical painrating scale.

\section{Clinical tests}

Six active movement-control tests were derived from descriptions by Comerford and Mottram [28,29]. These tests were Bent knee fall-out (BKFO; Figure 1), Standing bow (SB; Figure 2), Single leg small knee bend + lungelean (SLKB+LL; Figure 3), Double leg lift-lower (DLL-L; Figure 4), Double leg lift-alternate leg extension (DLLALE; Figure 5) and Double straight leg lower (DSLL; Figure 6). These tests, used in clinical treatment of the studied population, were initially selected based on discussions with clinicians with fairly long experiences of various clinical assessments in marines. Further, they are suggested for use with Swedish marines to screen and evaluate movement control of the back and hip. In principal, the tests were designed to evaluate the subjects' ability to control or prevent defined movements of the lumbar spine and hip, while performing specific movement

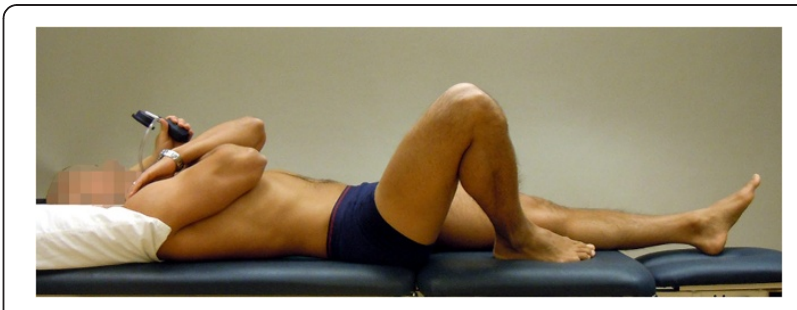

Figure 1 The bent knee fall-out (BKFO) test (reproduced with permission from Movement Performance Solutions). The BKFO test was used to test the ability to prevent rotation of the lumbar spine during abduction/lateral rotation (bent knee fall-out) of the hip. The test was classified as a low-load (threshold) test. Photos illustrates examples of views during the test. Start position: The subject lay supine with both legs straight. A pressure biofeedback unit (Chattanooga Group, Hixon, TN) was positioned between the lumbar lordosis and bench ipsilaterally to the test side and inflated to a pressure of $40 \mathrm{mmHg}$. A folded towel (towel thickness adjusted to match the height of the biofeedback unit) was placed between lumbar lordosis and bench on the contralateral side. The subject then flexed the hip and knee on the side to be tested until the foot was in line with the contralateral knee. Lumbar position was corrected (if needed) so that the biofeedback unit was $40 \mathrm{mmHg}$. Test movement: While preventing the lumbar spine from rotating, the subject lowered the bent leg out to the side, to $45^{\circ}$ abduction/ lateral rotation (bent knee fall-out) and then returned to the starting position. Criteria: Pass: $\leq 4 \mathrm{mmHg}$ away from the initiating $40 \mathrm{mmHg}$ during bent knee fall out.

in the lower extremity. Based on clinical/empirical knowledge with marines, we think that the loading complexity of the hip/lower back created by the active movement component in the tests adequately challenged fieldobserved weak links in marines' musculoskeletal system. For this reason these tests were performed in functionrelated positions/situations that adequately challenged these critical points of the musculoskeletal system. Three tests were classified as low-threshold (Figures 1,2,3) and three as high-threshold (Figures 4,5,6) [17], based on muscle activation levels/recruitment of slow- (low-threshold) and fast-twitch motor units (high-threshold) [30]. Two tests included movement control of both back and hip (Figures 3, 5) and four included movement control of the back (Figures 1, 2, 4, 6). Four tests were performed supine (Figures 1, 4, 5, 6) and two standing (Figures 2, 3). Initially a seventh test were included, "plank and twist" [28], but due to uncertainty over the rating criteria (not clearly observed during pre-study training) this test was early excluded. In four of the tests (Figures 1,4, 5,6) an air-filled pressure sensor (Pressure Biofeedback Unit, Chattanooga Group, Hixon, TN) was used to monitor lumbar movement. This sensor was originally developed for monitoring such spinal movement in clinical tests [31,32] and has proved useful for evaluating lumbar-muscle control [14-16] and neck-muscle function [33]. 


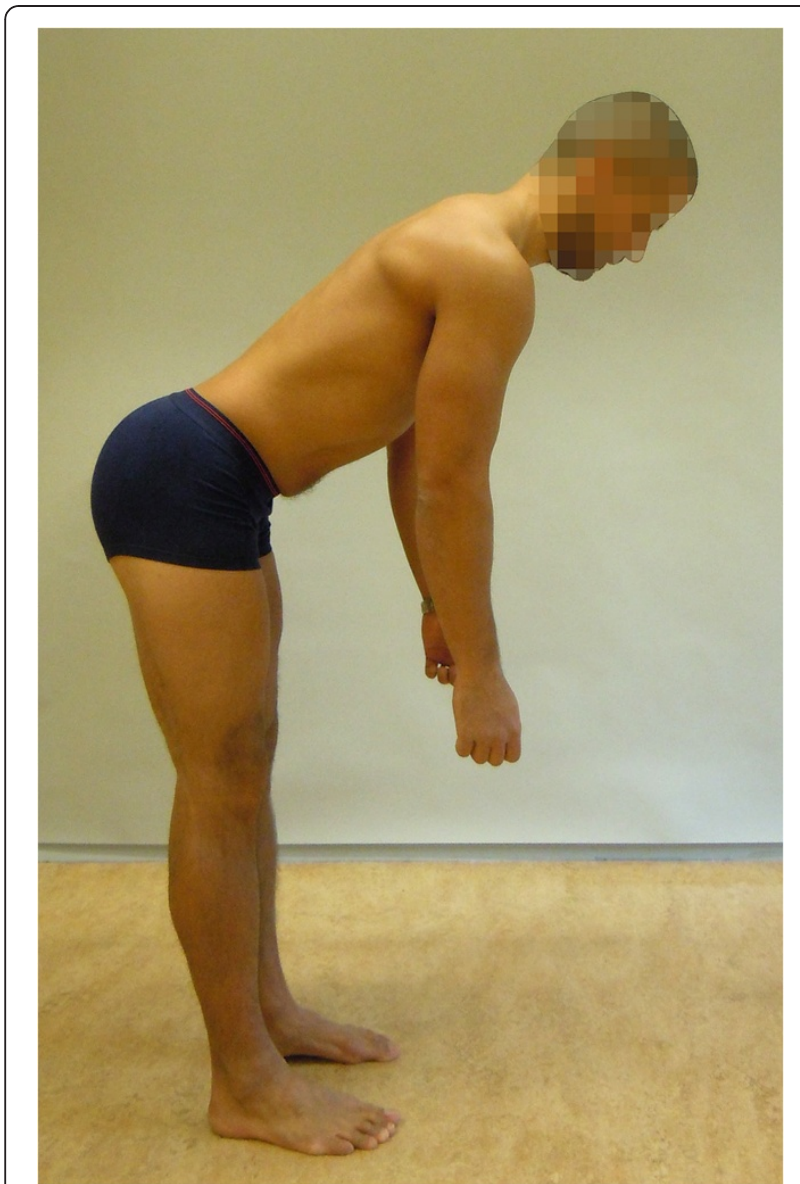

Figure 2 The Standing bow (SB) test (reproduced with permission from Movement Performance Solutions). The SB test was used to test the ability to prevent flexion of the lumbar spine during defined movement of the hip in upright standing. The test was classified as a low threshold test. Photos illustrates examples of views during the test. Start position: The subject stood with the pelvis relaxed and lumbar spine in neutral position. Test movement: While keeping the spine straight (not letting it round out or over arch) the subject bent the hips forwards to $50^{\circ}$ (forward lean). Criteria: Pass: forward lean with maintenance of lumbar spine in a neutral position.

\section{Test protocol}

After filling in initial questionnaires, each subject was instructed to wear only underwear so that movements of the lumbar spine, hips and lower extremities could be properly observed. The tests were performed in a standardized order (as specified from Figures 1, 2, 3, 4, 5, 6). The observers instructed every other enrolled subject as scheduled for test one (randomized for first subject), and this order was kept in the re-testing procedure. At first, the subjects received standardized instructions orally for the test and the instructing observer also demonstrated the test (see Additional file 1 for examples). All subjects then performed one trial with feedback and, if needed, received further visual, oral and/or manual instructions/

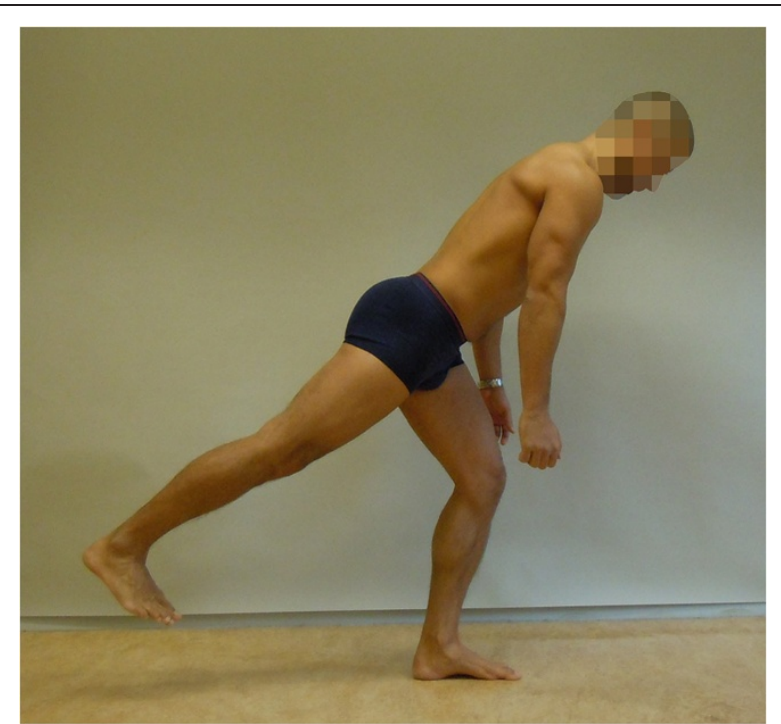

Figure 3 The Single leg small knee bend+lunge-lean (SLKB+LL) test (reproduced with permission from Movement Performance Solutions). The SLKB+LL test was used to test the ability to prevent motion of the lumbar spine and control movement of the hips during a lunge and forward-lean of the trunk, performed in a single leg knee-bend position. The test was classified as a low threshold test. Photos illustrates examples of views during the test. Start position: The subject stood with one foot back and the other in front (two feet lengths) from the rear foot. With the pelvis facing straight ahead and while keeping the back upright, the subject bent the knee to a forward lunge onto the front foot, allowing the rear heel to lift. Test movement: While keeping the spine straight, pelvis and chest facing ahead and knee and thigh over second toe, the subject bent forward at the hips to $45^{\circ}$ forward leaning. The subject then lifted the rear foot off the floor and kept the leg extended, in a straight line with the body. This position was held for $5 \mathrm{~s}$. Criteria: Pass: $5 \mathrm{~s}$ holding the position of $45^{\circ}$ forward lean over the front foot with the rear leg extended in line with the trunk, while maintaining the lumbar spine in a neutral position and without changing pelvis or hip position.

guidance by the instructing observer. This was done in order to ensure full understanding of the test performance and to ensure that the test result did not reflect the subject's unfamiliarity with the movement. Subjects then performed the tests and the performance was assessed simultaneously by the two observers using a standardized assessment rating protocol (see Additional file 1 for details) and then dichotomized (i.e. "fail" if one direction in any region was uncontrolled). The test protocol took approximately 30 minutes to conduct. To familiarize themselves with the test procedure and protocol, the observers trained on in vivo observations on a total of nine subjects together, dispersed over two occasions before study start. Here, the observers discussed and synchronized test performance, instructions and rating procedure. During the testing, however, there was no such communication, and the observers were blinded to each other's scores. 


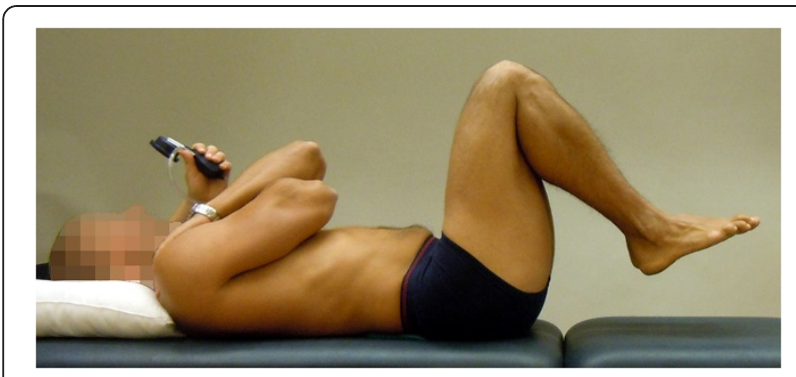

Figure 4 The Double leg lift-lower (DLL-L) test (reproduced with permission from Movement Performance Solutions). The DLL-L test was used to test the ability to prevent extension and flexion of the lumbar spine during defined movement of the hip, performed supine. The test was classified as a high threshold test Photos illustrates examples of views during the test. Start position: The subject lay in crook lying ( $45^{\circ}$ hip flexion), knees and feet together with arms folded across chest. A pressure biofeedback unit (Chattanooga Group, Hixon, TN) was positioned between the lumbar lordosis and bench and inflated to a pressure of $40 \mathrm{mmHg}$. Test movement: While preventing the lumbar spine from moving, the subject lifted both feet off the bench to $90^{\circ}$ hip flexion and then returned to the starting position. Criteria: Pass: $<5 \mathrm{mmHg}$ away from the initiating $40 \mathrm{mmHg}$ while no movement in lumbar spine.

\section{Analysis}

Data on inter- and intra-observer agreement was determined by calculating kappa coefficients, 95\% confidence intervals (CI) and percentage agreement. The kappa coefficient is the quantity of agreement, adjusted for

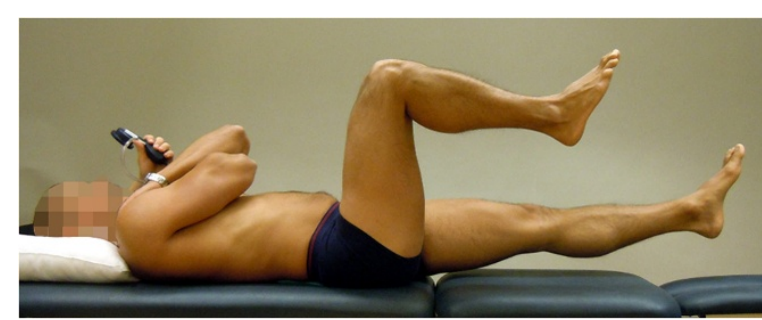

Figure 5 The Double leg lift-alternate leg extension (DLL-ALE) test (reproduced with permission from Movement Performance Solutions). The DLL-ALE test was used to test the ability to prevent extension, flexion and rotation of the lumbar spine, as well as leg abduction, lateral rotation and hip forward glide, during defined movement of the leg performed supine. The test was classified as a high threshold test. Photos illustrates examples of views during the test. Start position: The subject lay in crook lying ( $45^{\circ}$ hip flexion), knees and feet together with the arms folded across the chest. A pressure biofeedback unit (Chattanooga Group, Hixon, TN) was positioned between the lumbar lordosis and bench, and inflated to a pressure of $40 \mathrm{mmHg}$. Test movement: While preventing the lumbar spine from moving (monitored with pressure biofeedback unit and visually), the subject lifted both feet off the bench to $90^{\circ}$ hip flexion, then lowered and straightened one leg to fully extended position, and then back to $90^{\circ}$ hip flexion. This movement was then repeated with the other leg, and both legs were then finally returned to starting position. Criteria: Pass: $<5 \mathrm{mmHg}$ away from $40 \mathrm{mmHg}$ while no movement in lumbar spine. The extending leg was not to move away from the midline or turnout.

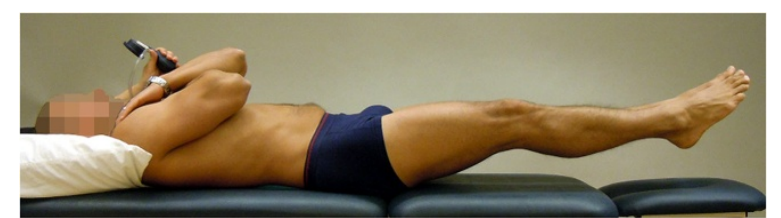

Figure 6 The Double straight leg lower (DSLL) test (reproduced with permission from Movement Performance Solutions). The DSLL test was used to test the ability to prevent extension and flexion of the lumbar spine during defined movement of the legs, performed supine. The test was classified as a high threshold test. Photos illustrates examples of views during the test. Start position; The subject lay in crook position ( $45^{\circ}$ hip flexion), knees and feet together with arms folded across chest. A pressure biofeedback unit (Chattanooga Group, Hixon, TN) was positioned between the lumbar lordosis and bench and inflated to a pressure of $40 \mathrm{mmHg}$. Test movement; While preventing the lumbar spine from moving (monitored with pressure biofeedback unit) the subject lifted both feet off the bench to $90^{\circ}$ hip flexion, then lowered and straightened both legs to fully extended, and then back to $90^{\circ}$ hip flexion. Criteria: Pass: $<5 \mathrm{mmHg}$ away from $40 \mathrm{mmHg}$ while no movement in lumbar spine

chance $[19,34]$. It has a maximum of 1.0, indicating perfect agreement, while zero indicates agreement no better than chance [19]. When interpreting the strength of agreement for the kappa coefficient, we used the standards proposed by Landis and Koch [35], where a kappa coefficient was: $0=$ poor, $0.01-0.20=$ slight, $0.21-0.40=$ fair, $0.41-0.60=$ moderate, $0.61-0.80=$ substantial, and $0.81-1=$ almost perfect. These standards were applied to the mean value from tests 1 and 2 (inter-observer reliability) and from test-re-test reproducibility (intra-observer reliability). To account for sampling error, the lower 95\% $\mathrm{CI}$ of the obtained value of kappa coefficient should be $>0.2$ [19]. The percentage agreement was calculated by dividing the numbers of agreed observations with the total number of observations for each test. The finitepopulation correction factor (fpc) [36] was applied to correct the variance on account of the sample size (n) in relation to the population $(N)$ :

$$
\mathrm{fpc}=(\sqrt{ }[(N-n) /(N-1)] \times \mathrm{SE})
$$

To investigate the discriminative validity of the bestfitting test/s for rated back- and lower-extremity pain during the previous six months and at present, respectively, the Akaike information criterion (AIC) autoregression was used on initial data (test 1 ). Hence, the lower the AIC value generated, the better the fit of the model:

$$
\mathrm{AIC}=2 k-2 L,
$$

where $\mathrm{k}$ is the number of variables (or tests) in the statistical model and L is the log likelihood of the model. A 
regression model with more than three variables/tests or a sensitivity less than $60 \%$ test was considered to be of limited value. A subject with both back- and lowerextremity pain was analysed in both groups. To check for systematic differences between tests one and two (systematic bias), McNemar analyses were applied with a $\mathrm{p}$ value of 0.05 as significant.

\section{Results}

One subject was lost to follow-up and one subject elected not to complete the follow up DSLL test due to recurrent back pain. Twenty-three subjects (70\%) rated that they had had back and/or lower extremity pain the previous six months, and eleven subjects (33\%) rated that they had ongoing back and/or lower extremity pain (see Table 1 for details).

\section{Inter-observer reliability}

Table 2 shows kappa coefficients (к) and 95\% confidence intervals representing inter- observer reliability. Three tests, BKFO ( $\left.\kappa_{\text {test } 1}=0.89 ; \kappa_{\text {test } 2}=1.00\right)$, DLL-L $\left(\kappa_{\text {test } 1}=\right.$ $\left.0.87 ; \kappa_{\text {test } 2}=0.93\right)$ and DLL-ALE $\left(\kappa_{\text {test } 1}=0.84 ; \kappa_{\text {test } 2}=\right.$ $0.87)$, reached almost- perfect inter-observer reliability with mean $\mathrm{K}$-coefficients from tests 1 and $2>0.86$. Results for two of the remaining tests, SLKB+LL $\left(\kappa_{\text {test } 1}=\right.$ $\left.0.60 ; \kappa_{\text {test } 2}=0.63\right)$ and DSLL ( $\left.\kappa_{\text {test } 1}=0.53 ; \kappa_{\text {test } 2}=0.81\right)$, showed substantial inter-observer reliability with mean $\mathrm{K}$ coefficients $>0.61$, while for SB $\left(\kappa_{\text {test } 1}=0.49 ; \kappa_{\text {test } 2}=\right.$ $0.63)$ the inter-observer reliability was moderate with mean $\mathrm{k}$-coefficients of 0.56 . A lower value of the $95 \%$ $\mathrm{CI}>0.2$, representing both first and second testing, was noted for all tests except DSLL and SLKB+LL.

\section{Intra-observer reliability}

Table 3 presents kappa coefficients and 95\% confidence intervals for intra-observer reliability. Three tests, BKFO $\left(\kappa_{\text {obs. } \mathrm{A}}=0.64 ; \kappa_{\text {obs. } \mathrm{B}}=0.53\right), \mathrm{SB}\left(\kappa_{\text {obs. } \mathrm{A}}=0.48 ; \kappa_{\text {obs. } \mathrm{B}}=\right.$ $0.39)$, and DLL-L $\left(\kappa_{\text {obs. }}\right.$ A $=0.63 ; \kappa_{\text {obs. }}$ B $\left.=0.40\right)$ had moderate intra-observer reliability with mean $\mathrm{k}$-coefficients of 0.44-0.58. The remaining three tests, SLKB+LL ( $\kappa_{\text {obs. }}$ $\left.\mathrm{A}=0.43 ; \kappa_{\text {obs. } \mathrm{B}}=0.31\right)$, DLL-ALE $\left(\kappa_{\text {obs. } \mathrm{A}}=0.39 ; \kappa_{\text {obs. } \mathrm{B}}=\right.$ 0.21 ), and DSLL ( $\kappa_{\text {obs. }} \mathrm{A}=0.24 ; \kappa_{\text {obs. }}$ в $=0.32$ ) had fair intra-observer reliability with mean $\mathrm{K}$-coefficients of 0.22 0.37 . For observer A, lower 95\% CI exceeded 0.2 in three tests (BKFO, SB and DLL-L), while observer B ended with a lower $95 \% \mathrm{CI}$ of less than 0.2 for all tests. The McNemar analysis showed that significantly more subjects passed the DLL-ALE test on re-testing than at the initial testing procedure (Table 4). This was significant for both observers $(p=0.026$ and $p=0.003)$. For observer $B$, this was also significant $(p=0.026)$ for the DLL-L test.

\section{Sensitivity/specificity}

Table 5 shows a discriminative summary of the bestfitting test(s) for back and lower-extremity pain rated for the previous six months and at present, respectively. For observer $\mathrm{A}$, the best fitting tests were BKFO and DSLL $(\mathrm{AIC}=41.8,94 \% / 47 \%$ (sens/spec) $)$ for back pain the previous six months while, for observer $\mathrm{B}$, a five-variable model $(\mathrm{AIC}=42.9,89 \% / 67 \%($ sens $/ \mathrm{spec})$ ) including BKFO and DSLL emerged as best fitting tests for back pain the previous six months. However, when including only BKFO and DSLL for observer B, sensitivity/specificity were reduced by six and seven percent, respectively $(\mathrm{AIC}=44.2,83 \% / 60 \%$ (sens/spec) $)$. For both observers, the BKFO and DSLL model discriminated prior back

Table 1 Rated back and lower-extremity (LE) pain the previous six months and at present $(\mathbf{n}=\mathbf{3 3})$

\begin{tabular}{|c|c|c|c|c|c|}
\hline \multirow[t]{3}{*}{ Ratings } & \multicolumn{3}{|c|}{ Pain previous six months } & \multicolumn{2}{|c|}{ Pain at present } \\
\hline & \multirow[b]{2}{*}{$n^{c}(\%)$} & \multirow{2}{*}{$\frac{\text { Worst pain }^{\mathrm{a}}}{\text { md (range) }}$} & \multirow{2}{*}{$\frac{\text { Average pain }^{\mathrm{a}}}{\text { md (range) }}$} & \multirow[b]{2}{*}{$n^{c}(\%)$} & \multirow{2}{*}{$\frac{\text { Pain at present }}{\text { md (range) }}$} \\
\hline & & & & & \\
\hline No pain & $10(30)$ & & & $22(67)$ & \\
\hline Back pain ${ }^{\mathrm{b}}$ & $18(55)$ & & & $9(27)$ & \\
\hline Thoracic pain & $12(36)$ & $5(1-7)$ & $2(1-7)$ & $5(15)$ & $1(1-5)$ \\
\hline Lumbar pain & $15(45)$ & $3(2-10)$ & $1(1-6)$ & $7(21)$ & $2(1-6)$ \\
\hline LE pain ${ }^{\mathrm{b}}$ & $16(48)$ & & & $8(24)$ & \\
\hline Hip/Thigh pain & $2(6)$ & $2(2-2)$ & $1(1-1)$ & $0(0)$ & $0(0)$ \\
\hline Knee pain & $14(42)$ & $3(1-8)$ & $2(1-5)$ & $6(18)$ & $3(1-4)$ \\
\hline Foot/ankle pain & $5(15)$ & $7(2-8)$ & $3(1-5)$ & $3(9)$ & $3(2-7)$ \\
\hline Only Back pain & $7(21)$ & & & $3(9)$ & \\
\hline Only LE pain & $5(15)$ & & & $2(6)$ & \\
\hline Back and LE pain ${ }^{\mathrm{b}}$ & $11(33)$ & & & $6(18)$ & \\
\hline
\end{tabular}

${ }^{\text {aPain }}$ rated on numeric rating scale, NRS.

${ }^{b}$ Subjects could rate pain in more than one region.

${ }^{c}$ Number of subjects that rated $\geq 1$ on numeric pain ratings the previous six months and at present, respectively. 
Table 2 Inter-observer reliability: kappa coefficient, 95\% confidence intervals, percent agreement and standard error

\begin{tabular}{|c|c|c|c|c|c|c|}
\hline Test & BKFO & SB & SLKB+LL & DLL-L & DLL-ALE & DSLL \\
\hline \multicolumn{7}{|l|}{ Test 1} \\
\hline Kappa coefficient (Cl 95\%) & $0.89(0.70-1.00)$ & $0.49(0.21-0.77)$ & $0.60(0.27-0.92)$ & $0.87(0.63-1.00)$ & $0.84(0.63-1.00)$ & $0.53(0.16-0.90)$ \\
\hline$\%$ agreement & 97.0 & 75.8 & 87.8 & 90.9 & 93.9 & 87.9 \\
\hline Std error & 0.107 & 0.153 & 0.180 & 0.101 & 0.111 & 0.203 \\
\hline \multicolumn{7}{|l|}{ Test 2} \\
\hline Kappa coefficient (Cl 95\%) & $1(1.00-1.00)$ & $0.63(0.39-0.87)$ & $0.63(0.19-1.00)$ & $0.93(0.80-1.00)$ & $0.87(0.72-1.00)$ & $0.81(0.58-1.00)$ \\
\hline$\%$ agreement & 100.0 & 81.3 & 93.8 & 96.9 & 93.8 & 93.6 \\
\hline Std error & 0 & 0.132 & 0.240 & 0.070 & 0.086 & 0.126 \\
\hline Mean kappa coefficient ${ }^{a}$ & 0.95 & 0.56 & 0.61 & 0.87 & 0.86 & 0.67 \\
\hline
\end{tabular}

Abbreviations: BKFO, Bent knee fall-out; SB, Standing bow; SLKB+LL, Single leg small knee bend + lunge-lean; DLL-L Double leg lift-lower; DLL-ALE, Double leg liftalternate leg extension; DSLL, Double straight leg lower; $\mathrm{Cl} 95 \%, 95 \%$ confidence intervals; Std error, standard error.

${ }^{a}$ Mean value of kappa coefficient obtained from test 1 and test 2.

pain if the BKFO test was passed and the DSLL test failed. Regarding lower-extremity pain, results for observer A revealed that the best-fitting tests for pain the previous six months were DLL-L and BKFO $(\mathrm{AIC}=45.2$, $81 \% / 59 \%$ (sens/spec)). Here, the model discriminated prior lower extremity pain not only in cases failing both tests, but also in those passing both tests. However, for observer B, DLL-L and SLKB+LL (AIC = 45.7, 44\%/88\% (sens/spec)) best predicted lower-extremity pain the previous six months, i.e. when passing DLL-L and failing SLKB+LL. A combination of low- and high-threshold tests consistently emerged as having the best fit. However, no model was sensitive for discriminating back and lower-extremity pain at present (sensitivity <60\%) (Table 5).

\section{Discussion}

We sought to determine the inter- and intra-observer reliability of six clinical tests targeted for screening and following marines' ability to perform accurate movement control. The tests had moderate-to-almost-perfect interobserver reliability while intra observer reliability was fair-to-moderate. Discriminative regression revealed that combinations of low- and high-threshold tests had discriminative validity for previous back pain, but were inconclusive for lower-extremity pain.

Since the recruited marines were on active duty, and not recruited from subjects seeking care, the external validity extends only to a population of marines on active service. This was felt to be a strength of the study since the selected tests were intended and limited for this operational group. The results could however be of interest for researchers and clinicians alike, particularly for those working with similar military units. Further, we used an in-vivo study procedure similar to our clinical or preventive work in respect of settings and rating criteria, hence strengthening the ecological validity of the study protocol. Here, also, a large number of military personnel are usually tested and screened in a short time frame, and we therefore applied one practice round for each test. Some of our tests included sub-scores on observations and ratings for several body regions $(\mathrm{SLKB}+\mathrm{LL}$ and DLL-ALE) and more than one direction of movement (SLKB+LL, DLL-L, DLL-ALE and DSLL). We believe,

Table 3 Intra-observer reliability: Kappa coefficient, 95\% confidence intervals, percent agreement and standard error

\begin{tabular}{|c|c|c|c|c|c|c|}
\hline Test & BKFO & SB & SLKB+LL & DLL-L & DLL-ALE & DSLL \\
\hline \multicolumn{7}{|l|}{ Observer A } \\
\hline Kappa coefficient (Cl 95\%) & $0.64(0.21-1.00)$ & $0.48(0.20-0.77)$ & $0.43(0.01-0.86)$ & $0.63(0.40-0.86)$ & $0.39(0.14-0.65)$ & $0.24(0-0.61)$ \\
\hline$\%$ agreement & 93.8 & 75.0 & 87.5 & 81.3 & 68.8 & 78.1 \\
\hline Std error & 0.232 & 0.156 & 0.232 & 0.126 & 0.140 & 0.203 \\
\hline \multicolumn{7}{|l|}{ Observer B } \\
\hline Kappa coefficient (Cl 95\%) & $0.53(0.11-0.95)$ & $0.39(0.10-0.67)$ & $0.31(0-0.68)$ & $0.40(0.15-0.66)$ & $0.21(0-0.43)$ & $0.32(0-0.69)$ \\
\hline$\%$ agreement & 90.6 & 68.8 & 81.3 & 68.8 & 57.6 & 78.1 \\
\hline Std error & 0.228 & 0.157 & 0.203 & 0.138 & 0.120 & 0.202 \\
\hline Mean kappa coefficient ${ }^{a}$ & 0.58 & 0.44 & 0.37 & 0.52 & 0.30 & 0.22 \\
\hline
\end{tabular}

Abbreviations: BKFO, Bent knee fall-out; SB, Standing bow; SLKB+LL, Single leg small knee bend + lunge-lean; DLL-L Double leg lift-lower; DLL-ALE, Double leg liftalternate leg extension; DSLL, Double straight leg lower; CI 95\%, 95\% confidence intervals; Std error, standard error.

${ }^{a}$ Mean value of kappa coefficient obtained from observer A and B. 
Table 4 Test results presented for each observer/test and number of cases identified that failed the test

\begin{tabular}{|c|c|c|c|c|}
\hline & \multirow{2}{*}{$\begin{array}{c}\text { Test } 1 \\
\text { Pass/fail }\end{array}$} & \multirow{2}{*}{$\begin{array}{c}\text { Test } 2 \\
\text { Pass/fail }\end{array}$} & \multicolumn{2}{|c|}{ Number of cases identified at test one with pain, that failed the test } \\
\hline & & & Back pain prev. $6 \mathrm{mo} / \mathrm{at}$ present & LE pain prev. $6 \mathrm{mo} / \mathrm{at}$ present \\
\hline \multicolumn{5}{|l|}{ Observer A } \\
\hline BKFO & $28 / 5$ & $30 / 2$ & $1 / 1$ & $4 / 1$ \\
\hline SB & $12 / 21$ & $14 / 18$ & $12 / 7$ & $9 / 6$ \\
\hline $\mathrm{SLKB}+\mathrm{LL}$ & $5 / 28$ & $3 / 29$ & $17 / 9$ & $14 / 8$ \\
\hline DLL-L & $15 / 18$ & $21 / 11$ & $9 / 4$ & $7 / 3$ \\
\hline DLL-ALE & $9 / 24$ & $17 / 15$ & $14 / 6$ & $11 / 7$ \\
\hline DSLL & $4 / 29$ & $7 / 24$ & $18 / 9$ & $14 / 8$ \\
\hline \multicolumn{5}{|l|}{ Observer B } \\
\hline BKFO & $27 / 6$ & $30 / 2$ & $1 / 1$ & $4 / 1$ \\
\hline SB & $14 / 19$ & $18 / 14$ & $11 / 7$ & $8 / 6$ \\
\hline $\mathrm{SLKB}+\mathrm{LL}$ & $7 / 26$ & $3 / 29$ & $15 / 8$ & $14 / 7$ \\
\hline DLL-L & $14 / 19$ & $22 / 10$ & $11 / 6$ & $7 / 5$ \\
\hline DLL-ALE & $7 / 26$ & 19/13 & $15 / 7$ & $12 / 8$ \\
\hline DSLL & $6 / 27$ & $7 / 24$ & $16 / 7$ & $13 / 8$ \\
\hline
\end{tabular}

Abbreviations: LE, lower extremity; BKFO, Bent knee fall-out; SB, Standing bow; SLKB+LL, Single leg small knee bend + lunge-lean; DLL-L Double leg lift-lower; DLLALE, Double leg lift -alternate leg extension; DSLL, Double straight leg lower.

however, that when applying clinical tests for screening purposes, for logistical reasons an overall screening examination should be used, for example, to set priorities for further individual clinical action, but also to collect data for epidemiological analyses and follow-up. Notably, the procedure with two observers scoring the same subject simultaneously, here with one observer instructing the subject, limits the inter-observer reliability to test performance only. Considering our discriminative regression, the number of subjects was rather small $(n=33)$, and this was why we pooled lumbar and thoracic pain as back pain, and hip/thigh, knee, ankle/foot pain as lower-extremity pain, respectively, in this analysis. Further, for defining pain at present, a cut-off of $\geq 1$ NRS may seem low.

Table 5 Discriminative analysis: Akaike information criterion (AIC), p-value and sensitivity/specificity of model variables for pain ratings

\begin{tabular}{|c|c|c|c|c|c|c|c|c|}
\hline & \multicolumn{5}{|l|}{ Model } & \multicolumn{3}{|c|}{ Discriminative analysis } \\
\hline & Var. 1 & Var. 2 & Var. 3 & Var. 4 & Var. 5 & $\mathrm{AIC}^{\mathrm{a}}$ & $p$ value & Sens/spec (\%) \\
\hline \multicolumn{9}{|l|}{ Back pain } \\
\hline \multicolumn{9}{|l|}{ prev. 6 month } \\
\hline Observer A & BKFO & DSLL & & & & 41.84 & 0.008 & $94 / 47$ \\
\hline Observer B & BKFO & SB & SLKB+LL & DSLL & DLL-ALE & 42.89 & 0.012 & $89 / 67$ \\
\hline \multicolumn{9}{|l|}{ at present } \\
\hline Observer A & SB & SLKB+LL & DLL-L & & & 36.68 & 0.046 & $33 / 92$ \\
\hline Observer B & SB & & & & & 40.49 & 0.140 & $0 / 100$ \\
\hline \multicolumn{9}{|l|}{ LE pain } \\
\hline \multicolumn{9}{|l|}{ prev. 6 month } \\
\hline Observer A & BKFO & DLL-L & & & & 45.24 & 0.040 & $81 / 59$ \\
\hline Observer B & $S L K B+L L$ & DLL-L & & & & 45.73 & 0.050 & $44 / 88$ \\
\hline \multicolumn{9}{|l|}{ at present } \\
\hline Observer A & SLKB+LL & DLL-L & DLL-ALE & & & 36.10 & 0.038 & $50 / 92$ \\
\hline Observer B & DLL-ALE & & & & & 36.10 & 0.034 & $0 / 100$ \\
\hline
\end{tabular}

Abbreviations: Sens, sensitivity; spec, specificity; LE, lower extremity; BKFO, Bent knee fall out; SB, Standing bow; SLKB+LL, Single leg small knee bend + lungelean; DLL-L Double leg lift-lower; DLL-ALE, Double leg lift -alternate leg extension; DSLL, Double straight leg lower.

${ }^{a} \mathrm{AIC}$, Akaike information criterion $=2 \mathrm{k}-2 \mathrm{~L}$ ( $\mathrm{k}=$ number of variables; $\mathrm{L}=\log$ likelihood). 
However, our experience with marines is that they underestimate their level of pain, also learned in other groups [37], and therefore a cut-off point of 1 , which equals "any pain experience", was selected. In addition, a study on US Army soldiers [37] showed that the prevalence of back pain history may be underestimated in long-term recall surveys compared to monthly follow-ups. Developing a standardized operational definition to determine functional limitation, including pain ratings and pain interference with activity (operational efficiency), may improve the reliability of a future outcome construct in marines, thus possible improving its potential on discriminative and predictive validity.

Our data on inter-observer reliability ranged from moderate to almost perfect agreement (Table 2). While no such reliability data exist on movement control tests in marines, our results on agreement between observers are consistent with $[14,15,22]$, or somewhat better than $[21,23]$, most other reliability studies of movement control conducted in the civilian population. Here, Enoch et al. [14] and Roussel et al. [15] presented good (moderate-to-excellent) inter-observer reliability with their invivo collected data, though few tests were similar to ours in terms of test protocol (c.f. BKFO and SB). We believe, however, that our results indicate that the present six clinical tests are reliable for use in screening programs with multiple observers in marines.

Our results on intra-observer reliability were fair-tomoderate. Surprisingly few studies report on the intraobserver reproducibility of movement control tests, particularly since such clinical tests are commonly used for follow-up evaluation. The results of Luomajoki et al. [21] ranged from fair to excellent intra-observer reliability for ten movement-control tests. Two of our tests (c.f. BKFO and SB) were similar to theirs, though our corresponding kappa coefficients indicated lower reproducibility than theirs. However, their test-retest ratings were based on video recordings of one test occasion.

Interestingly, for two of the tests in the present study, i.e. DLL-L and DL-ALE, more subjects "passed" the retest procedure than on the initial test occasion (Table 4). Such results may reflect a learning effect of the tests themselves (or systematic bias), and can only be manifested using observation from repeated testing. This probably also applies to our lower kappa coefficients on these tests. There were no clear indicators for any specific test being more difficult to instruct or evaluate relating it to poor re-test reproducibility. Further, only one other study [14] of movement control tests discloses how many practice times the subjects were allowed for each test. However, their study design did not include test-retest measurements, thus no intra-reliability analyses. Even so, we believe that repeated practice rounds may reduce learning effect, thus influence test reproducibility positively. In addition, improvement on the repeated test emphasizes the importance of including within-subject variation in test-re-test data relevant for clinical interpretation. Future studies, however, need to consider a trade-off between "realistic" amount of practice rounds related to their clinical work and sufficient elimination of learning effects.

One of the tests, the SLKB+LL, showed substantial inter-observer reliability at the re-test, with a kappa coefficient of 0.63 , but with a percentage agreement as high as $94 \%$. This discrepancy was probably due to an uneven number that passed/failed the test (Table 4), and it demonstrates how the kappa coefficient could be affected by such prevalence [38]. In order to adjust prevalence effects on kappa values, different types of adjustment have been discussed [19]. For example, with the prevalence-adjusted bias-adjusted kappa (PABAK), the adjusted kappa may be calculated with a maintained level of agreement, hence creating a "hypothetical population" with optimal distribution of pass/fail ratio [39]. Such adjusted coefficients may indeed add to the understanding of external validity extended to other populations such as other military units or possibly in civilian contexts. However, prevalence effects on kappa coefficients are themselves informative in a particular population [40] and, within the present study aim, we elected to report conventional kappa only. Further, the SLKB $+\mathrm{LL}$ and the DSLL showed lower 95\% CIs of the kappa coefficient of less than 0.2 on one test occasion, respectively, thus indicating an increased risk of measurement error. For intra- observer reliability, this was also so for most of the tests for both observers, here probably affected by the present learning effects. This should be considered in follow-up evaluation and interpretation with the present clinical tests.

Regarding discriminative validity, our results indicate that combinations of low- and high- threshold movementcontrol tests had some discriminative validity for previous back pain, but not for present pain. Concerning lower extremity pain, there were differences in sensitivity/specificity between observer $\mathrm{A}$ and $\mathrm{B}$, also for tests included in best fitting model, thus limiting the discriminative power of these observations. While we have learned that the AIC auto-regression rather accurately separates tests that do not really relate/contain properties with the dependent variable, pre-selection of tests with good kappa-coefficients may have strengthened our regression model. However, we believe our discriminative findings are an important complement to pain ratings, particularly since altered motor control may persist after pain relief [11] and long-term recall of pain may be underestimated [37], as indicated above. Our results somewhat support the use and interpretation of test combinations, rather than information 
from single tests. Since the BKFO and DSLL model discriminated prior back pain if the BKFO (low-threshold test) was passed, and the DSLL (high-threshold test) failed, the clinical and physiological implication of such results should be further validated. Even so, this is interesting since Roussel et al. [15] showed that two movement-control tests could predict injury in the back or lower-extremity over six months in professional ballet dancers. However, within the limits of the present study, the direction of causality is uncertain. In other words, does the pain experience cause certain results with movement control or vice versa. Also, our results say little about future incidents, and we believe therefore that further research should address the predictive validity of movement-control tests for musculoskeletal disorders in marines. Such knowledge would certainly contribute to the evidence for use of such screening tests in this group of military personnel.

\section{Conclusions}

Clinical tests that emphasize movement control for back and hip had moderate-to-almost-perfect inter-observer reliability, indicating that these tests are reliable as screening tests using several observers with marines. However, testretest reproducibility was not as accurate, with intraobserver reliability ranging from fair to moderate. This should be considered in follow-up evaluation. Our results also indicated that combinations of low- and highthreshold movement-control tests had discriminative validity for earlier back pain, but were inconclusive for lower-extremity pain. Further studies should emphasize predictive validity with clinically convenient tests for musculoskeletal disorders among marines.

\section{Additional file}

Additional file 1: Appendix A. (Inter-and intra-observer reliability of clinical movement-control test for marines).

\section{Competing interests}

The authors declare that they have no competing interests.

\section{Authors' contributions}

AM participated in the conception and design of the study, acquisition, analysis and interpretation of the data and was the main writer of the paper. $\mathrm{JH}$ participated in the planning, acquisition and interpretation of the data as well as writing and revising the paper. KN was involved in the design of the study, acquisition of the data and revising the paper. BOÄ was the senior project researcher, participating in the conception and design of the study, analyzing and interpreting the data, and writing and revising the paper. All the authors read and approved the final manuscript.

\section{Acknowledgements}

Funding from the Swedish Armed Forces PhD programme and financial support from Svenska Militärläkarföreningen are gratefully acknowledged. We also thank the 1st Marine Regiment, Swedish Armed Forces, for funding and overall support, and we especially extend our thanks to the marines who participated. The funding organizations had no authority over or input into any part of the study. We would also like to thank Mark Comerford, Sarah
Mottram and Movement Performance Solutions for their help with test design and modification.

\section{Author details}

'Department of Neurobiology, Care Sciences and Society, Division of Physiotherapy, Karolinska Institutet, Huddinge, Sweden. ${ }^{2}$ Swedish Armed Forces, Regional Medical Service Mälardalen, Berga, Sweden. ${ }^{3}$ Fysiocenter Odenplan, Stockholm, Sweden. ${ }^{4}$ Swedish Armed Forces, 1st Marine Regiment, 2nd Amphibious Battalion, Berga, Sweden.

Received: 9 March 2012 Accepted: 23 December 2012

Published: 29 December 2012

\section{References}

1. Kaufman KR, Brodine S, Shaffer R: Military training-related injuries: surveillance, research, and prevention. Am J Prev Med 2000, 18:54-63.

2. Riddell $D$ : Changes in the incidence of medical conditions at the commando training centre, royal marines. J R Nav Med Serv 1990, 76:105

3. Linenger J, West L: Epidemiology of soft-tissue/musculoskeletal injury among US marine recruits undergoing basic training. Mil Med 1992, 157:491.

4. Hayton J: Reducing medical downgrading in a high readiness royal marine unit. JR Army Med Corps 2004, 150:164-167.

5. Skeehan CD, Tribble DR, Sanders JW, Putnam SD, Armstrong AW, Riddle MS: Nonbattle injury among deployed troops: an epidemiologic study. Mil Med 2009, 174:1256-1262.

6. Lincoln AE, Smith GS, Amoroso PJ, Bell NS: The natural history and risk factors of musculoskeletal conditions resulting in disability among US army personnel. Work 2002, 18:99-113.

7. Larsson H, Broman L, Harms-Ringdahl K: Individual risk factors associated with premature discharge from military service. Mil Med 2009, 174:9-20.

8. Larsson H, Larsson M, Sterberg H, Harms-Ringdahl K: Screening tests detect knee pain and predict discharge from military service. Mil Med 2008, 173:259-265.

9. Belmont PJ, Goodman GP, Waterman B, DeZee K, Burks R, Owens BD: Disease and nonbattle injuries sustained by a US army brigade combat team during operation iraqi freedom. Mil Med 2010, 175:469-476.

10. Cohen SP, Brown C, Kurihara C, Plunkett A, Nguyen C, Strassels SA: Diagnoses and factors associated with medical evacuation and return to duty for service members participating in operation Iraqi freedom or operation enduring freedom: a prospective cohort study. Lancet 2010, 375:301-309.

11. Hodges PW, Richardson CA: Inefficient muscular stabilization of the lumbar spine associated with low back pain: a motor control evaluation of transversus abdominis. Spine 1996, 21:2640.

12. Luomajoki H, Kool J, de Bruin ED, Airaksinen O: Movement control tests of the low back; evaluation of the difference between patients with low back pain and healthy controls. BMC Musculoskelet Disord 2008, 9:170.

13. Moseley GL, Hodges PW: Reduced variability of postural strategy prevents normalization of motor changes induced by back pain: a risk factor for chronic trouble? Behav Neurosci 2006, 120:474-476.

14. Enoch F, Kjaer P, Elkjaer A, Remvig L, Juul-Kristensen B: Inter-examiner reproducibility of tests for lumbar motor control. BMC Musculoskelet Disord 2011, 12:114.

15. Roussel NA, Nijs J, Mottram S, Van Moorsel A, Truijen S, Stassijns G: Altered lumbopelvic movement control but not generalized joint hypermobility is associated with increased injury in dancers. A prospective study. Man Ther 2009, 14:630-635.

16. Roussel N, Nijs J, Truijen S, Vervecken L, Mottram S, Stassijns G: Altered breathing patterns during lumbopelvic motor control tests in chronic low back pain: a case-control study. Eur Spine J 2009, 18:1066-1073.

17. Mottram S, Comerford M: A new perspective on risk assessment. Phys Ther Sport 2008, 9:40-51.

18. Stephens J, Usherwood T: The mechanical properties of human motor units with special reference to their fatiguability and recruitment threshold. Brain Res 1977, 125:91-97.

19. Sim J, Wright CC: The kappa statistic in reliability studies: use, interpretation, and sample size requirements. Phys Ther 2005, 85:257.

20. Bruton A, Conway J, Holgate S: Reliability: what is it, and how is it measured? Physiotherapy 2000, 86:94-99. 
21. Luomajoki H, Kool J, de Bruin ED, Airaksinen O: Reliability of movement control tests in the lumbar spine. BMC Musculoskelet Disord 2007, 8:90.

22. Murphy D, Byfield D, McCarthy P, Humphreys K, Gregory A, Rochon R: Interexaminer reliability of the Hip extension test for suspected impaired motor control of the lumbar spine. J Manipulative Physiol Ther 2006, 29:374-377.

23. Van Dillen LR, Sahrmann SA, Norton BJ, Caldwell CA, Fleming DA, McDonnell MK, Woolsey NB: Reliability of physical examination items used for classification of patients with low back pain. Phys Ther 1998, 78:979.

24. Norman GR: SD: biostatistics: The bare essentials. 3edth edition. London: McGraw-Hill Medical; 2008

25. Williamson A, Hoggart B: Pain: a review of three commonly used pain rating scales. J Clin Nurs 2005, 14:798-804.

26. Kuorinka I, Jonsson B, Kilbom A, Vinterberg H, Biering-Sørensen F, Andersson G, Jørgensen K: Standardised Nordic questionnaires for the analysis of musculoskeletal symptoms. Appl Ergon 1987, 18:233-237.

27. Von Korff M, Jensen MP, Karoly P: Assessing global pain severity by selfreport in clinical and health services research. Spine 2000, 25:3140-3151.

28. Comerford MJ: The performance matrix performance profiling, risk assessment \& training strategies for injury prevention \& performance enhancement. UK: KC International/Movement Performance Solutions: 2008.

29. Comerford MJ, Mottram SL: Diagnosis of uncontrolled movement, subgroup classification and motor control retraining of the lumbar spine. UK: KC International/Movement Performance Solutions; 2010.

30. Lieber R: Skeletal muscle structure, function, \& plasticity - The physiological basis of rehabilitation. 2nd edition. United States of America: Lippincott Williams \& Wilkins; 2002.

31. Jull GRC, Toppenberg R, Comerford M, Bui B: Towards a measurement of active muscle control for lumbar stabilisation. Aust J Physiother 1993, 39:187-193.

32. Richardson C, Jull G: Muscle control-pain control. What exercises would you prescribe? Man Ther 1995, 1:2-10.

33. Falla DL, Campbell CD, Fagan AE, Thompson DC, Jull GA: Relationship between cranio-cervical flexion range of motion and pressure change during the cranio-cervical flexion test. Man Ther 2003, 8:92-96.

34. Cohen J: A coefficient of agreement for nominal scales. Educ Psychol Meas 1960, 20:37-46.

35. Landis JR, Koch GG: The measurement of observer agreement for categorical data. Biometrics 1977, 33:159.

36. Birnbaum DP: Who is at risk of what? Infect Control Hosp Epidemiol 1999, 20:706-707.

37. Carragee EJ, Cohen SP: Lifetime asymptomatic for back pain: the validity of self-report measures in soldiers. Spine 2009, 34:978-983.

38. Feinstein AR, Cicchetti DV: High agreement but low kappa: I. The problems of two paradoxes* 1. J Clin Epidemiol 1990, 43:543-549.

39. Byrt T, Bishop J, Carlin JB: Bias, prevalence and kappa. J Clin Epidemiol 1993, 46:423-429.

40. Hoehler FK: Bias and prevalence effects on kappa viewed in terms of sensitivity and specificity. J Clin Epidemiol 2000, 53:499-503.

doi:10.1186/1471-2474-13-263

Cite this article as: Monnier et al: Inter- and intra-observer reliability of clinical movement-control tests for marines. BMC Musculoskeletal Disorders 2012 13:263.

\section{Submit your next manuscript to BioMed Central and take full advantage of:}

- Convenient online submission

- Thorough peer review

- No space constraints or color figure charges

- Immediate publication on acceptance

- Inclusion in PubMed, CAS, Scopus and Google Scholar

- Research which is freely available for redistribution 